\title{
Low-Level Laser Therapy Effects on Rat Blood Hemostasis Via Significant Alteration in Fibrinogen and Plasminogen Expression Level
}

\author{
Babak Arjmand $^{1}{ }^{\mathbb{D}}$, Mahmood khodadoost ${ }^{2}$, Somayeh Jahani Sherafat ${ }^{3}$, Mostafa Rezaei Tavirani $^{*}{ }^{*}$, \\ Nayebali Ahmadi ${ }^{4}$, Farshad Okhovatian ${ }^{5}$, Majid Rezaei Tavirani ${ }^{6}$ \\ ${ }^{1}$ Cell Therapy and Regenerative Medicine Research Center, Endocrinology and Metabolism Molecular-Cellular \\ Sciences Institute, Tehran University of Medical Sciences, Tehran, Iran \\ ${ }^{2}$ School of Traditional Medicine, Shahid Beheshti University of Medical Sciences, Tehran, Iran \\ ${ }^{3}$ Laser Application in Medical Sciences Research Center, Shahid Beheshti University of Medical Sciences, Tehran, \\ Iran \\ ${ }^{4}$ Proteomics Research Center, Faculty of Paramedical Sciences, Shahid Beheshti University of Medical Sciences, \\ Tehran, Iran \\ ${ }^{5}$ Physiotherapy Research Center, Shahid Beheshti University of Medical Sciences, Tehran, Iran \\ ${ }^{6}$ Faculty of Medicine, Iran University of Medical Sciences, Tehran, Iran
}

\author{
*Correspondence to \\ Mostafa Rezaei Tavirani, \\ Proteomics Research Center, \\ Faculty of Paramedical \\ Sciences, Shahid Beheshti \\ University of Medical \\ Sciences, Tehran, Iran \\ Email: tavirany@yahoo.com \\ Received: May 17, 2021 \\ Accepted: July 3, 2021 \\ Published online October 9, \\ 2021
}

\begin{abstract}
Introduction: There are many documents about the significant role of low-level laser therapy (LLLT) in different processes such as regenerator medicine and bone formation. The aim of this study is to assess the role of LLLT in blood hemostasis in rats via bioinformatic investigation.

Methods: The differentially expressed plasma proteins of treated rats via LLLT from the literature and the added 50 first neighbors were investigated via network analysis to find the critical dysregulated proteins and biological processes by using Cytoscape software, the STRING database, and ClueGO. Results: A scale-free network including 55 nodes was constructed from queried and added first neighbor proteins. Fibrinogen gamma, fibrinogen alpha, and plasminogen were highlighted as the central genes of the analyzed network. Fibrinolysis was determined as the main group of biological processes that were affected by LLLT.

Conclusion: Findings indicate that LLLT affects blood hemostasis which is an important point in approving the therapeutic application of LLLT and also in preventing its possible complication.

Keywords: Laser therapy; Differentially expressed proteins; Bioinformatics; Rat; Blood hemostasis.
\end{abstract}

\section{Introduction}

The application of a laser in different fields of medicine implies more investigation to discover its molecular mechanism and possible complication. ${ }^{1}$ Hemostasis as a process that is involved in the cessation of bleeding from a blood vessel is studied in the presence of many stress conditions. ${ }^{2-4}$ There are many documents about the molecular aspect of low-level laser therapy (LLLT), which are concerned with proteomics, genomics, and bioinformatics..$^{5-7}$ Since the high throughput methods provide large numbers of data, bioinformatics as a useful tool is applied to organize and interpret the findings to present a new concept. ${ }^{8}$

Network analysis as one of the bioinformatics fields is an approach that studies the relationship between the assessed data. In this mode of analysis, the large numbers of proteins can include in a unique interacted unit to form a scale-free network. In the scale-free networks, there are few nodes (proteins in this study) that play crucial roles in network construction. ${ }^{9}$ Centrality parameters of the studied nodes are important characters of the nodes of the network that rank the node as critical and usual nodes. Two significant central parameters are degree and betweenness centrality. The nodes that are characterized with high values of degree and betweenness centrality are known as hub and bottleneck nodes respectively. The common hubs and bottlenecks are hub-bottlenecks which are potent central elements of the analyzed network..$^{10-12}$ The central nodes of a network determine the possible function of the network. ${ }^{13,14}$

Gene ontology is a method that determines related biological processes, molecular functions, biochemical pathways, and cellular components for the studied genes. ${ }^{15,16}$ Results of gene ontology are used to assess the molecular mechanism of many diseases. ${ }^{17,18}$ In many cases, network analysis and gene ontology are tied together to

Please cite this article as follows: Arjmand B, khodadoost M, Jahani Sherafat S, Rezaei Tavirani M, Ahmadi N, Okhovatian F, Rezaei Tavirani M. Low-level laser therapy effects on rat blood hemostasis via significant alteration in fibrinogen and plasminogen expression level. J Lasers Med Sci. 2021;12:e59. doi:10.34172/jlms.2021.59. 
solve the complexity of the studied systems. ${ }^{19-21}$ In the present study, the limited numbers of dysregulated plasma proteins of rats that are treated via LLLT in interaction with the main numbers of the first neighbors are selected to be analyzed via network analysis. The central proteins are investigated via gene ontology to find the critical modulated biological processes after LLLT.

\section{Methods}

Six dysregulated rat plasma proteins after LLLT were extracted from the published data by Kilik et al. ${ }^{22}$ Based on the methods of this article, 8 male Wistar rats in two groups, control group (C) and irradiated group (I), were investigated to find the effect of LLLT. Radiation is administrated by the gallium-aluminum-arsenide (GaAlAs) diode laser (Maestro/CCM, Medicom Prague, Czech Republic, $\lambda=830 \mathrm{~nm}$, oval shape of beam-spot size $\star 1 \mathrm{~cm}^{2}$, power density $450 \mathrm{~mW} / \mathrm{cm}^{2}$, total daily dose 60,3 $\mathrm{J} / \mathrm{cm}^{2}$, irradiation time 134 seconds) via transcutaneous irradiation for 9 days. Plasma proteins were analyzed via two-dimensional gel electrophoresis and the digested spots were identified by MALDI-TOF mass spectrometry. Details of the procedure are described in the published article. $^{22}$ The queried differentially expressed proteins (DEPs) plus 50 first neighbors from the STRING database were analyzed by using Cytoscape software v3.7.2. The central nodes were assessed via gene ontology by ClueGO plugin of Cytoscape. The related significant biological processes were selected from "GO, Biological Process,
EBI, UniProt-GOA-ACAP-ARAP, 08.05.2020”.

\section{Results}

Six identified proteins including haptoglobin (HP), hemopexin (HPX), fibrinogen gamma (FGG), fetuin-A (FETUA), Fetuin-B (FETUB), and alpha-1-antitrypsin (A1AT) were extracted from the published data by Kilik et al. ${ }^{22}$ The first three proteins (HP, HPX, and FGG) are the up-regulated proteins while the other ones are deregulated individuals. Among the 6 queried differentially expressed genes (DEGs), A1AT was not recognized by the STRING database. The network including 55 nodes (five queried DEPs and 50 added first neighbors) and 1034 edges was created (Figure 1). The nodes of the network and the centrality parameters of the nodes are presented in Table 1. The last queried DEP is located in the row of 23 in Table 1 and its degree is 40 . The degree of 40 was selected as cutoff and the 24 nodes that were characterized by degree value above 40 were considered for more analysis. The results of gene ontology of the selected 24 proteins are presented in Table 2. 56 terms clustered in four classes of biological processes are presented in Table 2. Each one of the two first groups includes one term while the third group is characterized by 18 terms. The larger group contains 36 biological processes.

\section{Discussion}

As it is shown in Figure 1, the collection of queried DEPs and the added first neighbor proteins formed a scale-

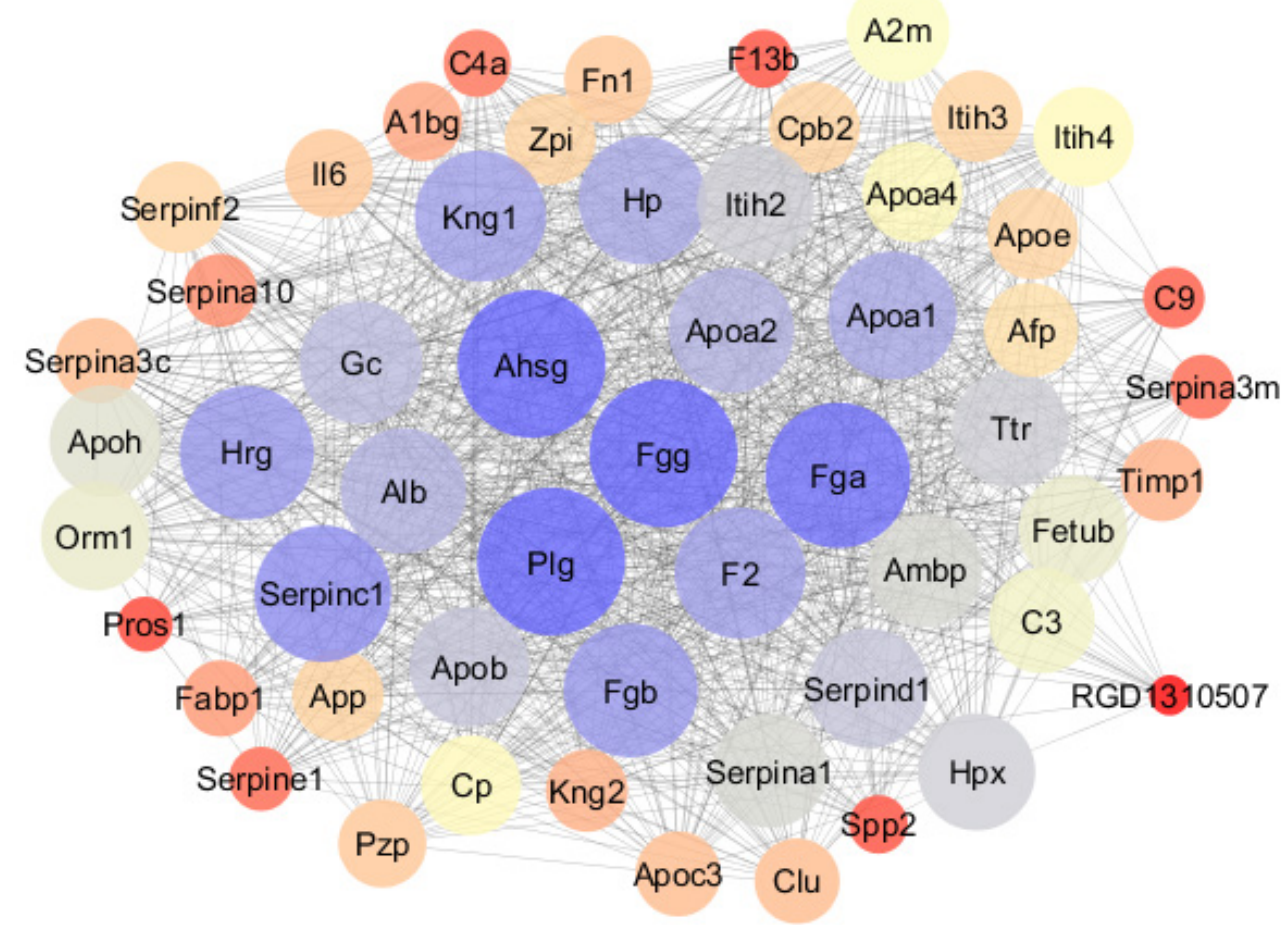

Figure 1. Network Including 5 Recognized Queried DEPs and 50 Added First Neighbors. The nodes are layout based on degree value. Red to blue color and increasing size of nodes refer to higher values of degree. 
Table 1. The 55 nodes of the analyzed network and the related centrality parameters are presented

\begin{tabular}{|c|c|c|c|c|c|c|}
\hline $\mathbf{R}$ & Display Name & Query Term & Degree & BC & $\mathrm{CC}$ & Stress \\
\hline 1 & Ahsg & FETUA & 54 & 0.017 & 1.000 & 902 \\
\hline 2 & Fgg & FGG & 54 & 0.017 & 1.000 & 902 \\
\hline 3 & Plg & & 54 & 0.017 & 1.000 & 902 \\
\hline 4 & Fga & & 53 & 0.015 & 0.982 & 828 \\
\hline 5 & Serpinc1 & & 50 & 0.013 & 0.931 & 694 \\
\hline 6 & Fgb & & 49 & 0.012 & 0.915 & 666 \\
\hline 7 & $\mathrm{Hrg}$ & & 49 & 0.013 & 0.915 & 692 \\
\hline 8 & $\mathrm{~F} 2$ & & 48 & 0.010 & 0.900 & 592 \\
\hline 9 & Kng1 & & 48 & 0.011 & 0.900 & 602 \\
\hline 10 & Apoa1 & & 47 & 0.009 & 0.885 & 540 \\
\hline 11 & $\mathrm{Hp}$ & $\mathrm{HP}$ & 47 & 0.011 & 0.885 & 584 \\
\hline 12 & Alb & & 46 & 0.009 & 0.871 & 510 \\
\hline 13 & Apoa2 & & 46 & 0.008 & 0.871 & 498 \\
\hline 14 & Gc & & 45 & 0.008 & 0.857 & 460 \\
\hline 15 & Apob & & 44 & 0.008 & 0.844 & 458 \\
\hline 16 & Serpind1 & & 44 & 0.008 & 0.844 & 476 \\
\hline 17 & Hpx & HPX & 43 & 0.007 & 0.831 & 434 \\
\hline 18 & Itih2 & & 43 & 0.008 & 0.831 & 452 \\
\hline 19 & Ttr & & 43 & 0.006 & 0.831 & 396 \\
\hline 20 & Ambp & & 42 & 0.006 & 0.818 & 348 \\
\hline 21 & Serpina1 & & 42 & 0.005 & 0.818 & 334 \\
\hline 22 & Apoh & & 41 & 0.005 & 0.806 & 314 \\
\hline 23 & Fetub & FETUB & 40 & 0.006 & 0.794 & 316 \\
\hline 24 & Orm1 & & 40 & 0.006 & 0.794 & 346 \\
\hline 25 & $\mathrm{C} 3$ & & 39 & 0.005 & 0.783 & 314 \\
\hline 26 & $\mathrm{~A} 2 \mathrm{~m}$ & & 38 & 0.005 & 0.771 & 292 \\
\hline 27 & Apoa4 & & 37 & 0.005 & 0.761 & 272 \\
\hline 28 & $\mathrm{Cp}$ & & 37 & 0.004 & 0.761 & 258 \\
\hline 29 & Itih4 & & 37 & 0.003 & 0.761 & 202 \\
\hline 30 & Afp & & 35 & 0.003 & 0.740 & 180 \\
\hline 31 & Apoe & & 34 & 0.003 & 0.730 & 180 \\
\hline 32 & App & & 34 & 0.003 & 0.730 & 198 \\
\hline 33 & Cpb2 & & 34 & 0.003 & 0.730 & 180 \\
\hline 34 & Itih3 & & 34 & 0.003 & 0.730 & 198 \\
\hline 35 & Serpinf2 & & 34 & 0.005 & 0.730 & 254 \\
\hline 36 & Zpi & & 34 & 0.004 & 0.730 & 224 \\
\hline 37 & Fn1 & & 33 & 0.003 & 0.720 & 188 \\
\hline 38 & 116 & & 33 & 0.003 & 0.720 & 170 \\
\hline 39 & Pzp & & 33 & 0.002 & 0.720 & 128 \\
\hline 40 & Apoc3 & & 32 & 0.001 & 0.711 & 104 \\
\hline 41 & Clu & & 32 & 0.004 & 0.711 & 226 \\
\hline 42 & Serpina3c & & 32 & 0.002 & 0.711 & 106 \\
\hline 43 & Kng2 & & 31 & 0.002 & 0.701 & 104 \\
\hline 44 & Timp1 & & 31 & 0.003 & 0.701 & 164 \\
\hline 45 & A1bg & & 30 & 0.004 & 0.692 & 204 \\
\hline 46 & Fabp1 & & 29 & 0.001 & 0.684 & 90 \\
\hline
\end{tabular}

\begin{tabular}{llllll}
47 & Serpina10 & 28 & 0.001 & 0.675 & 78 \\
48 & C4a & 26 & 0.001 & 0.659 & 76 \\
49 & Serpina3m & 25 & 0.001 & 0.651 & 96 \\
50 & Serpine1 & 25 & 0.002 & 0.651 & 100 \\
51 & C9 & 24 & 0.001 & 0.643 & 76 \\
52 & F13b & 23 & 0.001 & 0.635 & 54 \\
53 & Spp2 & 23 & 0.000 & 0.635 & 36 \\
54 & Pros1 & 22 & 0.001 & 0.628 & 58 \\
55 & RGD1310507 & 17 & 0.000 & 0.593 & 8 \\
\hline Note: & Three queried genes appear in the third column. & &
\end{tabular}

Note: Three queried genes appear in the third column.

free network. In the many published documents, the importance of the scale-free network in the interpretation of the molecular mechanism is emphasized. Centrality analysis revealed that there are numbers of critical nodes that can play a role as central nodes. As it is represented in Table 1, FETUA and FGG are the top nodes based on degree value and also betweenness centrality; therefore, these two queried DEPs can be considered as hubbottlenecks. Like FETUA and FGG, PLG and FGA appear as hub-bottleneck nodes (see Figure 1 and Table 1). Based on these results, it is possible that the two queried DEPs (FETUA and FGG) and also the two added first neighbors (PLG and FGA) be considered as the critical proteins which are dysregulated by laser irradiation.

As it is depicted in Table 2, the group of terms, which is categorized as "neutral lipid catabolic process" is not related to the central proteins. Then this group of terms was ignored from more analysis. FETUA (AHSG) is involved in the two terms of the top two groups of the term including "cysteine-type endopeptidase inhibitor activity" and "acute-phase response". FGG and PLG are involved in the 29 and 20 terms of the last group of terms respectively. FGA, the last central protein, is related to the 29 terms of the last group of terms.

It can be concluded that the critical group of terms is the group which is classed as "fibrinolysis" and the crucial proteins are FGG, FGA, and PLG. FGG is a queried upregulated DEP and FGA and PLG are two added first neighbors. Fibrinolysis is a significant term which effects on hemostatic balance. ${ }^{23}$ Fibrinolysis and platelet function are tied together to control blood coagulation. ${ }^{24} \mathrm{As}$ it can be seen in Table 2, most terms in the "fibrinolysis" group are characterized by platelet, coagulation, hemostasis, and fibrinolysis. The important role of fibrinogen in blood hemostasis and the pathological condition due to dysregulation of fibrinogen expression is described in many documents. Dysregulation of fibrinogen expression is highlighted in COVID-19 infection disease. ${ }^{25}$ Plasminogen is an inactive form of plasmin, the enzyme that degrades fibrin and activates matrix metalloproteinases, which leads to extracellular matrix degradation. ${ }^{26}$ The closed relationship between plasminogen and fibrinogen 
Table 2. Biological processes related to the 24 top nodes of the analyzed network

\begin{tabular}{|c|c|c|}
\hline GO Term & $\%$ AP & Associated protein found \\
\hline Cysteine-type endopeptidase inhibitor activity & 5.33 & [Ahsg, Fetub, Hrg, Kng1] \\
\hline Acute-phase response & 7.55 & [Ahsg, F2, Hp, Orm1] \\
\hline Regulation of plasma lipoprotein particle levels & 4.69 & [Apoa1, Apoa2, Apob] \\
\hline Plasma lipoprotein particle organization & 8.57 & [Apoa1, Apoa2, Apob] \\
\hline Plasma lipoprotein particle remodeling & 14.29 & [Apoa1, Apoa2, Apob] \\
\hline Protein-containing complex remodeling & 13.64 & [Apoa1, Apoa2, Apob] \\
\hline Regulation of lipid catabolic process & 4.62 & [Apoa1, Apoa2, Apoh] \\
\hline Protein-lipid complex subunit organization & 7.69 & [Apoa1, Apoa2, Apob] \\
\hline Neutral lipid catabolic process & 10.81 & [Apoa1, Apoa2, Apob, Apoh] \\
\hline Glycerolipid catabolic process & 6.35 & [Apoa1, Apoa2, Apob, Apoh] \\
\hline Positive regulation of lipid catabolic process & 10.00 & [Apoa1, Apoa2, Apoh] \\
\hline Intermembrane lipid transfer & 6.82 & [Apoa1, Apoa2, Apob] \\
\hline Protein-lipid complex remodeling & 14.29 & [Apoa1, Apoa2, Apob] \\
\hline Sterol transporter activity & 9.38 & [Apoa1, Apoa2, Apob] \\
\hline Acylglycerol catabolic process & 10.81 & [Apoa1, Apoa2, Apob, Apoh] \\
\hline Lipid transfer activity & 6.82 & [Apoa1, Apoa2, Apob] \\
\hline Triglyceride catabolic process & 11.54 & [Apoa1, Apob, Apoh] \\
\hline Cholesterol efflux & 6.25 & [Apoa1, Apoa2, Apob] \\
\hline Sterol transfer activity & 13.64 & [Apoa1, Apoa2, Apob] \\
\hline Cholesterol transfer activity & 14.29 & [Apoa1, Apoa2, Apob] \\
\hline Regulation of coagulation & 10.34 & [Apoh, F2, Fga, Fgb, Fgg, Hrg, Kng1, Plg, Serpinc1] \\
\hline Regulation of hemostasis & 10.71 & [Apoh, F2, Fga, Fgb, Fgg, Hrg, Kng1, Plg, Serpinc1] \\
\hline Protein activation cascade & 41.67 & [Apoh, Fga, Fgb, Fgg, Serpinc1] \\
\hline Blood coagulation & 5.78 & [Apoh, F2, Fga, Fgb, Fgg, Hrg, Kng1, Plg, Serpinc1, Serpind1] \\
\hline Hemostasis & 5.71 & [Apoh, F2, Fga, Fgb, Fgg, Hrg, Kng1, Plg, Serpinc1, Serpind1] \\
\hline Heterotypic cell-cell adhesion & 6.78 & [Apoa1, Fga, Fgb, Fgg] \\
\hline Negative regulation of coagulation & 15.09 & [Apoh, F2, Fga, Fgb, Fgg, Hrg, Kng1, Plg] \\
\hline Positive regulation of coagulation & 12.90 & [Apoh, F2, Hrg, Plg] \\
\hline Negative regulation of hemostasis & 15.38 & [Apoh, F2, Fga, Fgb, Fgg, Hrg, Kng1, Plg] \\
\hline Positive regulation of hemostasis & 14.29 & [Apoh, F2, Hrg, Plg] \\
\hline Platelet activation & 5.88 & [F2, Fga, Fgb, Fgg, Hrg] \\
\hline Blood coagulation, fibrin clot formation & 62.50 & [Apoh, Fga, Fgb, Fgg, Serpinc1] \\
\hline Regulation of response to wounding & 4.46 & [Apoh, F2, Fga, Fgb, Fgg, Hrg, Kng1, Plg, Serpinc1] \\
\hline Negative regulation of response to wounding & 8.33 & [Apoh, F2, Fga, Fgb, Fgg, Hrg, Kng1, Plg] \\
\hline Positive regulation of response to wounding & 4.60 & [Apoh, F2, Hrg, Plg] \\
\hline Regulation of blood coagulation & 10.84 & [Apoh, F2, Fga, Fgb, Fgg, Hrg, Kng1, Plg, Serpinc1] \\
\hline Platelet aggregation & 6.12 & [Fga, Fgb, Fgg] \\
\hline Positive regulation of blood coagulation & 14.29 & [Apoh, F2, Hrg, Plg] \\
\hline Negative regulation of blood coagulation & 15.69 & [Apoh, F2, Fga, Fgb, Fgg, Hrg, Kng1, Plg] \\
\hline Regulation of heterotypic cell-cell adhesion & 16.00 & [Apoa1, Fga, Fgb, Fgg] \\
\hline Regulation of wound healing & 5.66 & [Apoh, F2, Fga, Fgb, Fgg, Hrg, Kng1, Plg, Serpinc1] \\
\hline Negative regulation of wound healing & 10.26 & [Apoh, F2, Fga, Fgb, Fgg, Hrg, Kng1, Plg] \\
\hline Positive regulation of wound healing & 5.97 & [Apoh, F2, Hrg, Plg] \\
\hline Positive regulation of heterotypic cell-cell adhesion & 21.43 & [Fga, Fgb, Fgg] \\
\hline Endothelial cell apoptotic process & 5.08 & [Fga, Fgb, Fgg] \\
\hline Zymogen activation & 5.97 & [Apoh, Fga, Fgb, Fgg] \\
\hline
\end{tabular}


Table 2. Continued

\begin{tabular}{lcl}
\hline GO Term & $\%$ AP & Associated protein found \\
\hline Fibrinolysis & 31.82 & [Apoh, F2, Fga, Fgb, Fgg, Hrg, Plg] \\
Negative regulation of fibrinolysis & 42.86 & [Apoh, Hrg, Plg] \\
Positive regulation of vasoconstriction & 6.38 & [Fga, Fgb, Fgg] \\
Regulation of fibrinolysis & 27.27 & [Apoh, Hrg, Plg] \\
Negative regulation of epithelial cell apoptotic process & 5.56 & [Fga, Fgb, Fgg] \\
Plasminogen activation & 16.00 & [Apoh, Fga, Fgb, Fgg] \\
Regulation of extrinsic apoptotic signaling pathway via death domain receptors & 5.56 & [Fga, Fgb, Fgg] \\
Regulation of endothelial cell apoptotic process & 5.45 & [Fga, Fgb, Fgg] \\
$\begin{array}{l}\text { Negative regulation of extrinsic apoptotic signaling pathway via death domain } \\
\text { receptors }\end{array}$ & 10.00 & [Fga, Fgb, Fgg] \\
Negative regulation of endothelial cell apoptotic process & 9.38 & [Fga, Fgb, Fgg] \\
\hline
\end{tabular}

Note: Term $P$ value, term $P$ value corrected with Bonferroni step down, group $P$ value, and group $P$ value corrected with Bonferroni step down were less than 0.001. The four groups are shown in different colors and the name of the groups is bolded. \%AP refers to the percentage of associated proteins.

in body hemostasis was studied about 50 years ago and is a well-known association. ${ }^{27}$

Many effects such as wound healing, improving muscular function, effect on bacterial growth, effect on oral mucositis in cancer patients, bone formation, dentistry, and many other subjects are attributed to LLLT. $^{28-31}$ In the present study, a significant effect of LLLT on blood hemostasis is highlighted. This finding may be a crucial point in the application of LLLT as a therapeutic tool or in preventing the disadvantage of using LLLT.

\section{Conclusion}

It can be concluded that LLLT results in the modulation of body blood hemostasis. Fibrinogen and plasminogen were highlighted as the two important elements in this process.

\section{Ethical Considerations}

This project was approved by the ethical committee of Shahid Beheshti University of Medical Sciences. (code: IR.SBMU. RETECH.REC.1400.006)

\section{Conflict of Interests}

There is no conflict of interest.

\section{Acknowledgment}

This project is supported by Shahid Beheshti University of Medical Sciences.

\section{References}

1. Khalkhal E, Razzaghi M, Rostami-Nejad M, RezaeiTavirani M, Beigvand HH, Tavirani MR. Evaluation of laser effects on the human body after laser therapy. J Lasers Med Sci. 2020;11(1):91. doi: 10.15171/jlms.2020.15.

2. Smith SA, Travers RJ, Morrissey JH. How it all starts: Initiation of the clotting cascade. Crit Rev Biochem Mol Biol. 2015;50(4):326-36. doi: 10.3109/10409238.2015.1050550.

3. Austin AW, Wissmann T, von Känel R. Stress and hemostasis: an update. Semin Thromb Hemost 2013;39(08): 902-912. doi: 10.1055/s-0033-1357487
4. Raphael J, Mazer CD, Subramani S, Schroeder A, Abdalla M, Ferreira R, et al. Society of Cardiovascular Anesthesiologists clinical practice improvement advisory for management of perioperative bleeding and hemostasis in cardiac surgery patients. J Cardiothorac Vasc Anesth. 2019;33(11):2887-99. doi.10.1053/j.jvca.2019.04.003

5. Ogita M, Tsuchida S, Aoki A, Satoh M, Kado S, Sawabe M, et al. Increased cell proliferation and differential protein expression induced by low-level Er: YAG laser irradiation in human gingival fibroblasts: proteomic analysis. Lasers Med Sci. 2015;30(7):1855-66. doi: 10.1007/s10103-0141691-4.

6. Trajano L, Sergio L, Silva C, Carvalho L, Mencalha A, Stumbo A, et al. Low-level laser irradiation alters mRNA expression from genes involved in DNA repair and genomic stabilization in myoblasts. Laser Phys Lett. 2016;13(7):075601. doi:10.1088/1612-2011/13/7/075601

7. Arjmand B, Vafaee R, Razzaghi M, Rezaei-Tavirani M, Ahmadzadeh A, Rezaei-Tavirani S, et al. Central Proteins of Plasma in Response to Low-Level Laser Therapy Involve in Body Hemostasis and Wound Repair. J Lasers Med Sci. 2020;11(4 Suppl.):S55-S9. doi: 10.34172/jlms.2020.S9.

8. Zhong X-Z, Li X-X, Zeng Y, Wang S-P, Sun Z-Y, Tang Y-Q. Dynamic change of bacterial community during dairy manure composting process revealed by highthroughput sequencing and advanced bioinformatics tools. Bioresour. Technol. 2020;306:123091. doi: 10.1016/j. biortech.2020.123091

9. Azodi MZ, Arjmand B, Razzaghi M, Tavirani MR, Ahmadzadeh A, Rostaminejad M. Platelet and Haemostasis are the Main Targets in Severe Cases of COVID-19 Infection; a System Bioinformatics Study. Arch Acad Emerg Med. 2021;9(1):e27. doi: 10.22037/aaem.v9i1.1108

10. Wu J, Vallenius T, Ovaska K, Westermarck J, Mäkelä TP, Hautaniemi S. Integrated network analysis platform for protein-protein interactions. Nat Methods. 2009;6(1):75-7. doi: 10.1038/nmeth.1282.

11. Tsai C-J, Ma B, Nussinov R. Protein-protein interaction networks: how can a hub protein bind so many different partners?. Trends Biochem. Sci. 2009;34(12):594-600. doi: 10.1016/j.tibs.2009.07.007 
12. Safaei A, Tavirani MR, Oskouei AA, Azodi MZ, Mohebbi SR, Nikzamir AR. Protein-protein interaction network analysis of cirrhosis liver disease. Gastroenterol Hepatol Bed Bench. 2016;9(2):114-23. doi: 10.22037/ghfbb.v9i2.896

13. Wuchty S, Almaas E. Peeling the yeast protein network. Proteomics. 2005;5(2):444-9. doi: 10.1002/pmic.200400962.

14. Rabieian R, Abedi M, Gheisari Y. Central nodes in protein interaction networks drive critical functions in transforming growth factor beta-1 stimulated kidney cells. Cell J. 2017;18(4):514-531. doi: 10.22074/cellj.2017.4718

15. Harris MA, Clark J, Ireland A, Lomax J, Ashburner M, Foulger R, et al. Consortium GO. The Gene Ontology (GO) database and informatics resource. Nucleic acids Res. 2004;32(suppl_1):D258-D261. doi: 10.1093/nar/gkh036.

16. Ashburner M, Ball CA, Blake JA, Botstein D, Butler H, Cherry JM, et al. Gene ontology: tool for the unification of biology. Nat Genet. 2000;25(1):25-9. doi: 10.1038/75556.

17. Schlicker A, Lengauer T, Albrecht M. Improving disease gene prioritization using the semantic similarity of Gene Ontology terms. Bioinformatics. 2010;26(18):i561-i7. doi: 10.1093/bioinformatics/btq384.

18. Prakash $T$, Ramachandra NB. Integrated Network and Gene Ontology Analysis Identifies Key Genes and Pathways for Coronary Artery Diseases. Avicenna J Med Biotechnol. 2021;13(1):15-23. doi: 10.18502/ajmb.v13i1.4581

19. Ghatge M, Nair J, Sharma A, Vangala RK. Integrative gene ontology and network analysis of coronary artery disease associated genes suggests potential role of ErbB pathway gene EGFR. Mol Med Rep. 2018;17(3):4253-64. doi: 10.3892/mmr.2018.8393.

20. Rezaei Tavirani M, Mansouri V, Rezaei Tavirani S, Hesami Tackallou S, Rostami-Nejad M. Gliosarcoma proteinprotein interaction network analysis and gene ontology. Int J Cancer Manag. 2018;11(5): e65701. doi: 10.5812/ ijcm.65701.

21. Li L, Zhang N, Li S. Ranking effects of candidate drugs on biological process by integrating network analysis and Gene Ontology. Chinese Sci Bull. 2010;55(26):2974-80. doi: 10.1007/s11434-010-4067-6.
22. Kilik R, Bober P, Ropovik I, Beňačka R, Genči J, Nečas A, et al. Proteomic Analysis of Plasma Proteins after LowLevel Laser Therapy in Rats. Physiol Res. 2019;68(Suppl 4):S399-S404. doi: 10.33549/physiolres.934377.

23. Longstaff C. Measuring fibrinolysis: from research to routine diagnostic assays. J Thromb Haemost. 2018;16(4):652-62. doi: 10.1111/jth.13957

24. Chapin JC, Hajjar KA. Fibrinolysis and the control of blood coagulation. Blood rev. 2015;29(1):17-24. doi: 10.1016/j. blre.2014.09.003.

25. Rezaei-Tavirani M, Nejad MR, Arjmand B, Tavirani SR, Razzaghi M, Mansouri V. Fibrinogen Dysregulation is a Prominent Process in Fatal Conditions of COVID-19 Infection; a Proteomic Analysis. Arch Acad Emerg Med. 2021;9(1):e26. doi: 10.22037/aaem.v9i1.1128

26. Collen D. The plasminogen (fibrinolytic) system. Thromb. Haemost.1999;82(08):259-70.

27. Lucas MA, Fretto L, McKee P. The binding of human plasminogen to fibrin and fibrinogen. J Biol Chem. 1983;258(7):4249-56. doi:10.1016/s0021-9258(18)32614-0

28. Bjordal JM, Bensadoun R-J, Tunèr J, Frigo L, Gjerde K, Lopes-Martins RA. A systematic review with meta-analysis of the effect of low-level laser therapy (LLLT) in cancer therapy-induced oral mucositis. Support Care Cancer. 2011;19(8):1069-77. doi: 10.1007/s00520-011-1202-0.

29. De Marchi T, Junior ECPL, Bortoli C, Tomazoni SS, LopesMartins RÁB, Salvador M. Low-level laser therapy (LLLT) in human progressive-intensity running: effects on exercise performance, skeletal muscle status, and oxidative stress. Lasers Med Sci. 2012;27(1):231-6. doi: 10.1007/s10103011-0955-5.

30. Pinheiro AL, Oliveira MG, Martins PPM, Ramalho LMP, de Oliveira MAM, Júnior AN, et al. Biomodulatory effects of LLLT on bone regeneration. Laser ther. 2000;13(1):73-9. doi: 10.5978/islsm.13.73

31. Nussbaum EL, Lilge L, Mazzulli T. Effects of low-level laser therapy (LLLT) of $810 \mathrm{~nm}$ upon in vitro growth of bacteria: relevance of irradiance and radiant exposure. J clin laser med surg. 2003;21(5):283-90. doi: 10.15171/jlms.2020.15. 